\section{Research News}

\section{RNAi advances to mammals}

A powerful method to target genes for de-activation has gotten a new boost. RNAi (RNA-mediated interference) entered the spotlight in the mid-1990s with the discovery that double-stranded RNAs (dsRNAs) selectively inhibited gene expression in the nematode Caenorhabditis elegans. In C. elegans, inhibition persists throughout life and is heritable. Applying the technique to mammalian cells has been tricky, though - dsRNAs trigger an anti-viral system that non-specifically shuts off gene expression and induces cell death. Investigators have gotten around this by using dsRNAs that are too small to turn on anti-viral responses, about 20 bases. But such RNAs lose their potency within a week of application to human cells. Another hurdle has been getting such small sequences expressed intracellularly, a step towards long-term gene inactivation. Now, two groups describe new vector systems to stably express small RNAs in mammalian cells, Patrick Paddison et al. in the 15 April Genes and Development and Thijn Brummelkamp et al. in the 21 March online edition of Science. The two systems act differently but achieve the same end: efficient, specific downregulation of gene expression that persists through generations of cell division in cultured mammalian cells. The new research broadens the possibilities both for basic research and for the development of new therapies for human disease.

\section{Colon cancer - silenced no more}

Chromatin remodeling genes have been gaining in popularity lately as tumor-suppressor candidates. Now, add one more to the list: the SWI/SNF family member HTLF (helicase-like transcription factor). Helen Moinova et al. report in the 2 April Proceedings of the National Academy of Sciences that HTLF appears to act as a tumor suppressor for colon cancer-and that instead of being mutated in cancers, it's silenced by DNA methylation. Such silencing was detected in $43 \%$ of colon cancers, and in colon cancer cell lines as well. In cell lines, demethylating agents were able to restore $H T L F$ expression, and the transfected gene was able to suppress cell growth. The results add to speculation that the development of anti-cancer therapies based on demethylation could ultimately prove beneficial. (see p. 449)

\section{Parasite bites back}

People in malaria-stricken areas often come down with the disease repeatedly until they develop immunity. A new study in the April 15 Journal of Experimental Medicine suggests why: in mice, the malaria Plasmodium parasite (shown replicating in a red blood cell) appears to selectively destroy cells capable of specifically mediating long-term immunity against malaria-that is, Plas-

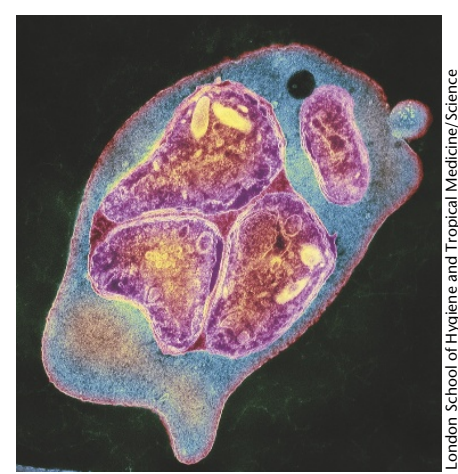
that target other agents, such as ovalbumin. Furthermore, experimental deletion of Plasmodium-specific CD4 ${ }^{+} \mathrm{T}$ cells results in an inhibited immune response upon re-infection. The researchers suspect that Plasmodium induces apoptosis of $\mathrm{CD}^{+} \mathrm{T}$ cells. They implicated interferon- $\gamma(\mathrm{IFN}-\gamma)$ in this killing process by treating mice with antiIFN- $\gamma$ antibodies and showing they interfered with Plasmodium induced $\mathrm{CD}^{+} \mathrm{T}$ cell demodium-targeted $\mathrm{CD}^{+}{ }^{+} \mathrm{T}$ cells. Huji Xu et pletion. A further understanding of how al. show that parasite infection results in the parasite kills $\mathrm{CD} 4^{+} \mathrm{T}$ cells could result depletion of $\mathrm{CD}^{+} \mathrm{T}$ cells directed against in the identification of new targets for a Plasmodium, but bypasses $\mathrm{CD}^{+} \mathrm{T}$ cells vaccine to counteract malaria.

\section{Breaking trail in spinal cord injury}

Spinal-cord injury paralyzes over 11,000 new people in accidents each year in the United States alone. These individuals have few treatment options, but a recent study provides a new direction for investigation. Elizabeth Bradbury et al. demonstrate that a bacterial enzyme that clips sugar groups off intracellular proteins can reduce symptoms of spinal cord injury in rats. The researchers tested the enzyme because they guessed it might clear the way for neurons to grow through the thicket of extracellular matrix that builds up around damaged tissue. The matrix is held together by a variety of molecules, including a class of proteins with proteoglycan sugar groups that are selectively removed by the bacterial enzyme. It turns out that the researchers had guessed correctly. In rats with much of their spinal cord severed and no use of their limbs, enzyme treatment resulted in several millimeters of nerve regrowth. That apparently was enough to restore some mobility to the rats, the authors report in the 11 April Nature. Enzyme-treated rats showed near-normal walking behavior on a grid and a beam, although they did not show recovery of finer sensory-locomotor tasks, such as the ability to peel off a piece of adhesive tape. The enzyme treatment could hold promise for clinical practice, especially in combination with other therapies.

\section{Putting the end on EBV}

Epstein-Barr virus (EBV) and other members of the herpesvirus family seem to live dangerously. They persist as extrachromosomal elements that must counteract nuclease degradation, chromosomal integration, DNA re-arrangement and other cellular assaults. These elements allow the viruses to take up long-term residence in human cells, where they replicate once per host cell division. What keeps the viral DNA intact? In the March Molecular Cell, Paul Lieberman and colleagues report that several proteins normally associated with human telomeres appear to shelter EBV viral DNA from damage. They identified the proteins in a complex with a viral protein that binds to the EBV origin of replication. The complex includes a telomere repeat-binding protein that protects against end-to-end chromosomal fusions (TRF2), another that may stabilize single-stranded DNA loops at telomeres (TRF1), and a telomere-specific poly-(ADP-ribose) polymerase (Tankyrase). These proteins associate with the origin-binding protein on DNA elements of the virus that resemble human telomeric repeats, and viruses that lacked these repeats seemed to be susceptible to damage. EBV infection is widespread in humans and is associated with certain types of cancers. Could the findings lead to new therapies for EBV and related viruses? That remains to be seen, but the new study certainly points the way.

By Charlotte Schubert \& Paul Morin 